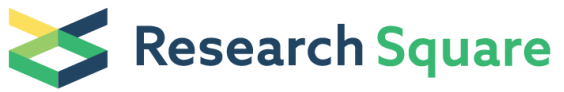 \\ Preprints are preliminary reports that have not undergone peer review. \\ They should not be considered conclusive, used to inform clinical practice, \\ or referenced by the media as validated information.
}

\section{Ultra-Conformal Skin Electrodes with Synergistically Enhanced Conductivity for Low-Motion Artifact Epidermal Electrophysiology}

\section{Yan Zhao}

Beijing Key Laboratory of Energy Conversion and Storage Materials, College of Chemistry, Beijing Normal University, Beijing 100875

\section{Song Zhang}

University of Southern Mississippi https://orcid.org/0000-0001-9815-7046

Tianhao Yu

Beijing Graphene Institute https://orcid.org/0000-0001-9637-352X

\section{Yan Zhang}

Beijing Key Laboratory of Energy Conversion and Storage Materials, College of Chemistry, Beijing Normal University, Beijing 100875

Guo Ye

Beijing Key Laboratory of Energy Conversion and Storage Materials, College of Chemistry, Beijing Normal University, Beijing 100875

Wenchao Jiang

Beijing Graphene Institute

Yifei Lu

Beijing Graphene Institute

\section{Weifeng Zhang}

Beijing Key Laboratory of Energy Conversion and Storage Materials, College of Chemistry, Beijing Normal University, Beijing 100875

\section{Xiaojia Du}

Beijing Key Laboratory of Energy Conversion and Storage Materials, College of Chemistry, Beijing Normal University, Beijing 100875

Jiakang Qiu

Beijing Key Laboratory of Energy Conversion and Storage Materials, College of Chemistry, Beijing Normal University, Beijing 100875

\section{Xiaodan Gu}

University of Southern Mississippi https://orcid.org/0000-0002-1123-3673

\section{Liu Nan ( $\square$ nanliu@bnu.edu.cn )}

Beijing Normal University

\section{Article}

Keywords: epidermal electronics, electrophysiology, dry electrode, graphene, PEDOT:PSS, transfer, $\pi-\pi$ interaction

Posted Date: August 27th, 2020

DOI: https://doi.org/10.21203/rs.3.rs-61379/v1

License: @ (1) This work is licensed under a Creative Commons Attribution 4.0 International License. Read Full License

Version of Record: A version of this preprint was published at Nature Communications on August 12th, 2021. See the published version at https://doi.org/10.1038/s41467-021-25152-y. 


\section{Abstract}

Accurate and imperceptible monitoring of electrophysiological signals is of primary importance for wearable healthcare. Stiff and bulky pregelled electrodes are now commonly used in clinical diagnosis, causing severe discomfort to users as well as artifact signals in motion. Here, we report a $100 \mathrm{~nm}$ ultrathin dry epidermal electrode that is able to conformably adhere to skin and accurately measure electrophysiological signals. This dry epidermal electrode was fabricated by direct transfer of Chemical Vapor Deposition (CVD) - grown graphene $(\sim 1 \mathrm{~nm})$ by poly (3,4-ethylenedioxythiophene) polystyrene sulfonate (PEDOT:PSS, 100 nm) as the transfer medium, namely PTG. It showed low sheet resistance $(45 \Omega / \mathrm{sq})$, high transparency $(80 \%)$, and mechano-electrical stability. The enhanced optoelectronic performance was due to the synergistic effect between graphene and PEDOT:PSS, which induced a high degree of molecular ordering on PEDOT and charge transfer on graphene by strong $\pi-\pi$ interaction. Together with ultra-thin nature, PTG exhibited a low electrochemical impedance when interfacing with skin, thereby leading to accurate electrophysiological signal detection with low motion artifact, as well as controlling robotic claws. This strategy to synergistically incorporate graphene and PEDOT:PSS together as ultra-conformal dry electrodes will have a significant impact on the development of epidermal electronics.

\section{Introduction}

Epidermal electronics refer to electronic devices that are mounted on the epidermis and able to acquire information about the body precisely or to provide therapy to the body. It can open new pathways for wearable healthcare ${ }^{1-3}$, athletic training, drug delivery ${ }^{4}$ systems, human-machine interaction ${ }^{5-7}$, etc. Skin-contact electrophysiology, such as electromyography (EMG), electrocardiography (ECG), and electroencephalogram (EEG), relying on the recording of biopotential, are important clinical measurements to assess health status. The ability to accurately and imperceptibly monitor electrophysiological signals is the most basic requirement for wearable electronics ${ }^{8}$. Skin-contact electrodes in clinical use are mainly pregelled $\mathrm{Ag} / \mathrm{AgCl}^{9-11}$. Although they provide good signal quality, the electrolytic gel may irritate the skin and will dry in a certain period, making it impossible for long-term monitoring and diagnosis. Moreover, with the gel interfacing on skin, electrode displacement with respect to skin will cause severe motion $\operatorname{artifact}^{12,13}$ and an electrical short circuit due to electrodes sticking together, resulting in poor electrophysiological signal resolution. To achieve high signal quality and feasible integration with other electronic components, ultra-conformal and dry electrodes are highly desirable for biopotential monitoring.

Conducting polymer ${ }^{14-18}$ and gold film ${ }^{13,17}$ are two frequently studied dry electrodes for electrophysiological recordings. Due to the low Young's modulus, conducting polymer, with a representative of PEDOT:PSS was recently proposed. PEDOT:PSS being fabricated into a soft 3D micropillar electrode array exhibited an intimate interface with cell, which could record action potentials from monolayer cell cultures ${ }^{19}$. Thin Au film has also emerged as a promising alternative because they are highly conductive and biocompatible. For example, Au film sandwiched between parylene film (sub-300 nm) was proved to be self-adhesive to the arbitrary 3D biological surface and able to monitor surface biopotentials with less motion-artifact ${ }^{2}$. However, both PEDOT:PSS and Au-film dry electrodes are expensive and visible on the skin, which may bring light-induced photoelectric artifact when applying them in certain scenarios. To make dry electrodes more compatibly integrated with other devices, low-cost and optical transparency are two essential characteristics to be introduced into dry electrodes for biopotential monitoring.

As the thinnest electrode material, graphene demonstrates superior optical transparency, high electrical conductivity, excellent mechanical property, and low electrochemical reactivity ${ }^{20-25}$. It is also regarded as a promising skin-electrode for electrophysiological signal detection ${ }^{8}$. Poly(methyl methacrylate) (PMMA) - assisted CVD graphene electronic tattoo, with a total thickness of $463 \pm 30 \mathrm{~nm}$, the optical transparency of $\sim 85 \%$, the sheet resistance of $1994.33 \pm 264 \Omega / \mathrm{sq}$ and stretchability of $\sim 40 \%$, was applied on skin to detect electrophysiological signals. To fabricate dry electrodes that are easy to handle and conformal to skin, low modulus polymer thin film (herein PMMA) was utilized. However, the PMMA polymer carrier layer will introduce contamination, cracks, or defects into the CVD-grown graphene during transfer, leading to a significant decrease in the conductivity of final electrodes ${ }^{26,27}$. Therefore, to improve the quality of electrophysiological recordings for graphene-based ultra-conformal dry electrodes, a modified PEDOT:PSS layer, not only capable of handling graphene as well as enhancing its superior electronic performances, was proposed in this work. 
Here, we elaborated a strategy for the fabrication of transparent, highly conductive, and ultrathin dry electrodes by using PEDOT:PSS thin film as a polymer carrier layer to handle and enhance the electrical performances of CVD-grown graphene for biopotential monitoring. To enable a thin, uniform, continuous and highly conductive PEDOT:PSS layer covered on top of graphene, surfactants and ionic compounds were added since pure PEDOT:PSS solution did not wet graphene and exhibited limited conductivity. Optimized dry electrodes of PEDOT:PSS transferred CVD graphene film (PTG) demonstrated a total thickness of $\sim 100 \mathrm{~nm}$, a sheet resistance of $\sim 45 \Omega / \mathrm{sq}$, the optical transparency of $\sim 80 \%$, and a decent electro-mechanical stability. The dramatic increase in electrical conductivity is not merely a consequence of a parallel connection of two conductive units, but also a synergistic effect of improved molecular packing of PEDOT and charge transfer on underlying graphene. This observation has been recognized by Grazing-incidence wide-angle x-ray scattering (GIWAXS), Raman, Electron spin resonance (ESR) spectroscopy, and so on. Our PTG electrode interfacing with skin showed 1.3 times decrease in electrochemical impedance compared with PMMA/graphene electronic tattoo, and thus a high signal-to-noise ratio for EMG and ECG recording. Our synergistic strategy towards ultra-conformal, transparent, and dry conductive electrodes will pave a new way for epidermal electronics.

\section{Results And Discussion}

Fabrication of PTG. To achieve dry electrodes with superior electronic performances for biopotential monitoring, we synthesized graphene via CVD ${ }^{28}$ method on copper foil and used conducting polymer PEDOT:PSS (Fig. 1a, 1b) as the carrier layer to transfer graphene. The fabrication process of PTG is presented in Fig. 1c. When pure PEDOT:PSS solution was spin-coated on graphene/Cu foil, it quickly de-wet, shrank into a droplet, and could not form a continuous transfer layer. This is because graphene is highly hydrophobic with a high contact angle $\left(96^{\circ}\right)$ due to the lack of oxygen-rich functional groups, which impedes PEDOT:PSS from wetting on the graphene (Figure S1). Adding surfactants ${ }^{29,30}$ is an efficient way to enhance the wettability of PEDOT:PSS solution as well as its electrical conductivity. Here, we chose sodium dodecyl sulfate (SDS) ${ }^{31}$, as it is a safe additive which has been widely used in detergents for laundry (Fig. 1b). In addition to SDS, an appropriate weight ratio of ionic compound ${ }^{32}$ (herein bis(trifluoromethane) sulfonimide lithium salt as an example, BSL in short), was also introduced into PEDOT:PSS solution to further improve the electrical conductivity and mechanical stretchabilitiy of PTG as skin electrodes. As-obtained PEDOT:PSS (with SDS and BSL)/graphene/Cu foil was annealed at $120^{\circ} \mathrm{C}$ for several minutes to enhance the interaction between PEDOT:PSS and graphene, and subsequently immersed in $\left(\mathrm{NH}_{4}\right)_{2} \mathrm{~S}_{2} \mathrm{O}_{8}$ solution to etch the underlying $\mathrm{Cu}$ foil, followed by rinsing in deionized water to remove possible residues (See Methods, Figure S2, S3). The resultant PTG thin film was then floated on water and scooped onto arbitrary substrates, such as a thin elastomer, glass, and even skin, working as a dry electrode immediately.

Optoelectronic performances of PTG. Due to the amphiphilic nature of these additives, the addition of SDS and BSL to PEDOT:PSS solution improves its wettability on the hydrophobic surface and helps to form a continuous and uniform PEDOT:PSS conductive layer on graphene. To verify the improvement in electrical conductivity of PTG with the addition of SDS and BSL, we measured 4probe sheet resistances and transmittances on a variety of PTGs as a function of SDS and BSL. The sheet resistance of PTG thin film was clearly reduced with increased SDS concentrations in the PEDOT:PSS solution (Fig. 2a). This is because the addition of SDS may weaken the electrostatic interaction between PEDOT and PSS, leading to the decoupling of PEDOT from PSS and increased crystallinity. In the meanwhile, transmittances of PTG increased with the addition of SDS, as SDS induced a less-viscous solution and a thinner PEDOT:PSS film was formed at the same spin-coating condition. When the concentration of the surfactant exceeded a critical micelle concentration, superfluous SDS may cause flocculation, even precipitation, which would generate rough surfaces and reduce the transmittance inevitably. We indeed observed that when the concentration of SDS was more than $1.0 \mathrm{wt} \%$, the PEDOT:PSS began to precipitate, resulting in big aggregates on PEDOT:PSS layer (Figure S4). Hence, the optimized concentration of SDS additive in PEDOT:PSS solution is chosen to be $1 \mathrm{wt} \%$. To achieve both high stretchability and conductivity, a small molecule ionic compound BSL was added as a second additive in PEDOT:PSS solution (1.0 wt\% SDS). As shown in Fig. 2b, with the increase of BSL concentration, the sheet resistance of PTG decreased, while the transmittances of PTG film initially increased at $1.0 \mathrm{wt} \% \mathrm{BSL}$ and then decreased due to the gelation of the PEDOT:PSS solution. To compromise the transparency and conductivity of PTG, BSL concentration was optimized as $1.0 \mathrm{wt} \%$, and the PTG film with monolayer graphene showed a sheet resistance as low as $45 \Omega$ /sq with the transmittance of $80 \%$ at $550 \mathrm{~nm}$ (Fig. $2 \mathrm{~b}$ ).

Next, the mechanoelectrical stability of PTG was evaluated (see Methods). We placed PTG onto poly(ethylene terephthalate) (PET) and bent it at various angles. Almost no resistance change was observed when PTG was at a bending radius from $12 \mathrm{~mm}$ to $2 \mathrm{~mm}$

(Fig. 2c). To evaluate its electrical durability at strain, PTG was then transferred onto an elastomeric styrene-ethylene-butadiene- 
styrene (SEBS) substrate, and the 2-probe resistance change was monitored under strain. It is noted that upon increasing the number of the underlying graphene layer, the resistance change becomes slower. For the SEBS-supported PEDOT:PSS-transferred tri-layer graphene, the resistance was kept as low as $1680 \Omega$ at $40 \%$ strain (Fig. 2d), suggesting that it is capable of working as a stretchable electrode up to $40 \%$ strain.

It is noteworthy that the sheet resistance of our CVD graphene is $~ 600 \Omega / s q$, while the PEDOT:PSS film enhanced by SDS and BSL at a thickness $\sim 100 \mathrm{~nm}$ shows a value of $\sim 1560 \Omega / \mathrm{sq}$ (Table S1). Upon connecting PEDOT:PSS and graphene in a parallel circuit, the parallel total resistance is calculated $433 \Omega / \mathrm{sq}$, which is almost 10 times higher of PTG $(45 \Omega / \mathrm{sq})$. This indicates that the synergistic enhancement between PEDOT: PSS and graphene is the main contributor to the high electrical conductivity of PTG. We hypothesized that by interfacing PEDOT:PSS with graphene, the molecular packing of PEDOT will be rearranged, with a highly ordered thin-film morphology as induced by the electron coupling from graphene. In the meantime, graphene will be charge doped by PEDOT:PSS while maintaining a clean interface without the removal of the polymer carrier layer (Fig. 2e).

Synergistic enhancement between PEDOT:PSS and graphene. To figure out the synergistic effect between PEDOT: PSS and graphene, we performed a comprehensive study on the structure of PTG. Raman spectroscopy is a powerful tool to characterize carbon-based materials. For the PEDOT:PSS film only, there was a vibration peak at $1434.5 \mathrm{~cm}^{-1}$ originated from C $=\mathrm{C}$ symmetrical stretching ${ }^{32}$ of PEDOT. After the addition of SDS and BSL, the peak position red-shifted to $1431.6 \mathrm{~cm}^{-1}$ and $1432.4 \mathrm{~cm}^{-1}$, respectively. The PEDOT chain in the pristine PEDOT:PSS aqueous solution is coiled due to the Coulombic interaction. With the addition of SDS and BSL, the coiled PEDOT chain becomes linear. The red-shift confirmed the structural transition of the thiophene ring on PEDOT chains, from a coil-like benzoid structure in pristine to a linear-like quinoid structure ${ }^{33,34}$ (Figure S5-S6). This structural transition led to an effective interchain coupling of PEDOT chains. After interfacing PEDOT:PSS with graphene (Fig. 3b), the $\mathrm{C}=\mathrm{C}$ symmetrical vibration peak of PTG further shifted to $1438.9 \mathrm{~cm}^{-1}$, indicating a more thorough structural transformation of PEDOT. Moreover, the $G$ band, a characteristic peak of CVD-grown graphene $\left(1586.4 \mathrm{~cm}^{-1}\right)$ was shifted to $1599.5 \mathrm{~cm}^{-1}$ in PTG. Such blue-shifts indicated that there is strong $\pi-\pi$ interaction between PEDOT:PSS layer and graphene, which will engender the delocalization of $\pi$ electrons, thus increasing the charge carrier mobility of PTG thin film.

The charge delocalization of $\pi$ electrons was also confirmed by UV-vis-NIR spectroscopy. Compared with pure PEDOT:PSS film, a strong absorbance occurred in PTG over a broad range, both at the 800-nm peak and the free-carrier tail of near-infrared region. This observation proved the charge delocalization between graphene and PEDOT:PSS, leading to the formation of polaron pairs, that is, bipolarons. The $\pi-\pi$ interaction effect was then studied by electron spin resonance (ESR), which probed the presence of unpaired electrons (Fig. 3d). The strong ESR signals of pure PEDOT:PSS films indicated the localized charge carrier in PEDOT chains. In contrast, at the presence of graphene, PTGs, either with SDS or BSL, showed significantly decreased ESR signals. This result implied that the charge carrier was converted from localized polaron states to delocalized bipolaron states upon interfacing PEDOT:PSS with graphene. Due to the strong $\pi-\pi$ interaction between PEDOT:PSS and the underlying graphene, a more ordered semicrystalline structure of PEDOT can be achieved, thus an enhanced electrical property of PTG can be obtained.

The effect of additives (SDS, BSL) and graphene on the molecular packing of PEDOT was further investigated by Grazing-incidence wide-angle x-ray scattering (GIWAXS), an advanced technique to analyze thin-film morphology. Figure 4a compared 2D GIWAXS patterns of pure PEDOT:PSS films and PTGs with and without additives, respectively. The 1D scattering profiles along $q_{z}$ and $q_{x y}$ directions were obtained from the 2D GIWAXS patterns (Figure S7). The (010) peak at around $q_{x y}=1.8 \AA^{-1}$ is attributed to the $\pi-\pi$ stacking of PEDOT, and their lattice spacings can be calculated from Bragg's law and shown as follows: $3.54 \AA$ (pure PEDOT:PSS), $3.46 \AA$ (PEDOT:PSS with SDS), $3.47 \AA$ (PEDOT:PSS with SDS and BSL) $3.40 \AA$ (PTG with SDS) and $3.37 \AA$ (PTG with SDS and BSL). With the addition of SDS, BSL, and mainly the underlying graphene, the $\pi-\pi$ stacking distance of PEDOT decreases from $3.54 \AA$ to $3.37 \AA$ (Fig. 4d), which ranked among the smallest intermolecular stacking distance of solution-processed PEDOT observed so far ${ }^{35}$. The closer packing of PEDOT chains in PTG again confirmed the strong $\pi-\pi$ interaction between graphene and PEDOT, which could contribute to an enhanced electrical conductivity.

The highly ordered molecular packing in PTGs can also be reflected in the surface morphology. We used atomic force microscopy (AFM) to observe the morphological evolution of pure PEDOT:PSS and PTGs (Fig. 4b). With the addition of SDS and BSL, limited change in the surface roughness and morphology can be observed when compared with pure PEDOT:PSS films. While interfacing with graphene, PTGs exhibited a distinct morphological transition from granules to nanofibrils, while a $2-3$ times increase in

Page $4 / 12$ 
roughness values was observed. This is consistent with a previous study, showing the granule-to-nanofibril transition when PEDOT became ordered ${ }^{32,35}$. The more ordered PEDOT packing in PTGs can be explained by the strong $\pi-\pi$ interaction between graphene and PEDOT:PSS. Overall, the synergistic enhancement between PEDOT:PSS and graphene will induce a higher crystallinity in PEDOT, so as to achieve a high conductivity for PTG to accurately detect electrophysiological signals.

Electrophysiological monitoring and robotic manipulating. Interfacial impedance is a critical parameter in epidermal electrophysiological measurement. For $\mathrm{Ag} / \mathrm{AgCl}$ electrodes, the wet conductive gel was used in skin/electrode interface, which inevitably introduced additional capacitance. Without the additional gel, PTG as dry electrodes would reduce the contact impedance. In addition, the thickness of PTG is about $100 \mathrm{~nm}$, the Young's modulus of our sample matches the stiffness of human stratum corneum ${ }^{36}$, which leads to intimate contact between electrodes and skin, resulting in lower impedance. The interfacial impedance analysis between electrodes and skin was conducted on the human arm with electrodes placed on the surface of it. As expected, PTG showed comparable interfacial impedance with skin as commercial $\mathrm{Ag} / \mathrm{AgCl}$ gel electrodes, and at $100 \mathrm{~Hz}$, the impedance $(\sim$ $32 \mathrm{k} \Omega)$ is lower than the $\mathrm{Ag} / \mathrm{AgCl}(\sim 45 \mathrm{k} \Omega)$, as shown in Fig. $5 \mathrm{a}$.

With low interfacial impedance, PTG were used in electrophysiological detection, with $\mathrm{Ag} / \mathrm{AgCl}$ as control. As shown in Fig. $5 \mathrm{~b}$, the amplitude of baseline noise in SEMG signals, is lower than $\mathrm{Ag} / \mathrm{AgCl}$ electrodes. The SNR values obtained by PTG (40.99 dB) is higher than $\mathrm{Ag} / \mathrm{AgCl}$ electrodes (39.02 dB) (Figure S8). As electrolytic gel will dry and induce electrode displacement, the major challenge of $\mathrm{Ag} / \mathrm{AgCl}$ electrodes in electrophysiological detection is the motion artifact. Hence the motion artifact was investigated here. We used electromechanical vibrator to monitor skin vibration in arm movement. The electromechanical vibrator was placed near the working electrodes (about $4 \mathrm{~cm}$ ) to induce skin vibration, which is similar to vibration in arm movement. The vibration was applied for $2 \mathrm{~s}$ and then rested for $2 \mathrm{~s}$. Figure $5 \mathrm{c}$ showed the sEMG noise of PTG and $\mathrm{Ag} / \mathrm{AgCl}$ electrode respectly. The average noise signal value of the PTG is just $13.7 \mu \mathrm{V}, 4.5$ times less than $75.7 \mu \mathrm{V}$ of $\mathrm{Ag} / \mathrm{AgCl}$ electrodes in the same condition. For ECG signals shown in Figure S9, all of the $\mathrm{P}, \mathrm{Q}, \mathrm{R}, \mathrm{S}$, and $\mathrm{T}$ waves can be clearly identified, and these characteristic peaks are crucial rhythmias-relevant parameters in clinical diagnosis. In addition, the infrared image (Figure S10) showed that a minimal level of heat was accumulated upon mounting the PTG electrodes on a volunteer's forearm, hence PTG is biocompatible. Our ultra-conformal PTG exhibited less motion artifact in sEMG and excellent ECG signal, demonstrating PTG is quite suitable for surface biopotential measurements.

The sEMG signals are generated by muscular excitation-contraction, and converting the sEMG signals to control artificial limb is urgently expected clinically. As shown in Fig. 5e, firstly the sEMG signals were extracted by our electrodes and then converted to Pulse Width Modulation (PWM) pulses to drive the servo motor. And it is notable that the pulses change is proportional to the muscle activities. Lastly, we demonstrated the manipulation of a mechanical claw (Movie S1). As shown in Fig. 5f, the open-close degree of the claw is related with the muscle activities. The claw is open when the the arm is at rest, and the claw gradually closes with the fist becomes tightened. Above demonstration indicated that our ultra-conformal and dry PTGs as skin electrodes could have potential applications in controlling artifact limbs in the future.

\section{Conclusion}

In conclusion, by leveraging two well-known skin electrodes together, we have demonstrated a transparent, highly conductive, and ultra-conformal dry electrode, which is PEDOT:PSS thin film-transferred CVD-grown graphene (PTG). Graphene transferred by PEDOT:PSS without the step of removing the carrier layer was confirmed to be electron doped with higher mobility; on the other hand, the PEDOT backbones showed improved molecular packing due to the underlying graphene. Thus, the synergistic enhancement effect between graphene and PEDOT:PSS resulted PTG with a very high conductivity ( $45 \Omega /$ sq @ $80 \%$ transmittance). Together with the ultrathin feature, PTG dry electrode exhibited excellent capability of electrophysiological signal recording, showing higher signal-to-noise ratio and less motion artifact than commercial $\mathrm{Ag} / \mathrm{AgCl}$ gel electrode, and being able to remotely control the robotic claws. Our proposed ultrathin, transparent and conductive dry electrodes will pave a new way for epidermal electronics. Further efforts will be working on patterning PTGs into multielectrode arrays for long-term, accurate and imperceptible monitoring of electrophysiological signals.

\section{Experimental Section}

\section{Materials and methods:}


PEDOT:PSS solution (Clevios PH 1000) was purchased from Han Feng, the sodium dodecyl sulfate (99\%) was obtained from Innochem, and the polyethylene oxide were obtained from Aladdin Industrial Corporation.

Fabrication of PTG dry electrodes: Cu foil was put in the quartz tube under a constant pressure of $20 \mathrm{sccm}$ (standard cubic centimeter per minute) $\mathrm{H}_{2}$ and $35 \mathrm{sccm} \mathrm{CH}$. After 40 minutes' growth at $1000{ }^{\circ} \mathrm{C}$, both sides of $\mathrm{Cu}$ foil were covered with monolayer graphene.The SDS of $0.25 \mathrm{wt} \%-1.5 \mathrm{wt} \%$ was added to PEDOT:PSS solution and stirred vigorously for $2 \mathrm{~h}$. The BSL was then added to PEDOT:PSS/SDS solution and stirred for another $2 \mathrm{~h}$. Before spin-coating, the prepared PEDOT:PSS solution was filtered by 0.45 $\mu \mathrm{m}$ PES syringe filter. And the PEDOT: PSS solution was spin-coated on the Cu foil with graphene at $2000 \mathrm{rpm}$ for $60 \mathrm{~s}$, forming a PEDOT:PSS/graphene/Cu structure. The PEDOT:PSS coated graphene on Cu foil was annealed at $100^{\circ} \mathrm{C}$. Subsequently, Cu foil was etched by ammonium persulfate $\left[\left(\mathrm{NH}_{4}\right)_{2} \mathrm{~S}_{2} \mathrm{O}_{8}\right]$ solution about $2 \mathrm{~h}$, then the PEDOT:PSS/graphene film was washed in deionized water for 3 times, The film can be transferred to arbitary substrates, such as silicon wafer, glass or flexible substrates.

sEMG and SECG measurements: Two working electrodes were placed on forearm, and the reference electrode was placed on the back of wrist for SEMG. The electrodes were connected $w$ aith commercial signal recording equipment (DueLite) and sEMG signals were digitally filtered in MATLAB by a six-order Butterworth filter. And the low cut-off frequency is $10 \mathrm{~Hz}$ while the high cut-off frequency is $450 \mathrm{~Hz}$. For ECG, two working electrodes and reference electrodes were placed on arm by using the Einthoven triangle principle. For easy handling, SEBS and tattoo paper were sometimes assisted as an additional substrate.

Motion artifact characterization: The electromechanical vibrator was placed near the working electrodes (about $4 \mathrm{~cm}$ ) to induce skin vibration, which is similar to vibration in arm movement. The vibration was supplied for about $2 \mathrm{~s}$ and then rested for another $2 \mathrm{~s}$.

\section{PTG Characterization:}

The sheet resistances were measured using a four-probe resistance measuring meter, with a Keithley 2400 Source Measure Unit. The transparency of the thin film was tested by an ultraviolet-visible spectrometer (UV-2450). The AFM images were obtained in tapping mode (Nanoscope III, Digital Instrument). The Grazing-incidence wide-angle x-ray scattering (GIWAXS) measurement was performed on a laboratory beamline system (Xenocs Inc. Xeuss 2.0) with an X-ray wavelength of $1.54 \AA$ and sample to detector distance of 17.1 $\mathrm{cm}$. An incidence angle of $0.2^{\circ}$ was used. Diffraction images were recorded on a Pilatus $1 \mathrm{M}$ detector (Dectris Inc.) and processed using the Nika software package, in combination with WAXS Tools. The Raman spectra were obtained at $532 \mathrm{~nm}$ excitation (LabRAM HR Evolution). The electrochemical impedance spectroscopy (EIS) measurements were conducted using a CHI600e electrochemical workstation (CH Instruments).

\section{Declarations}

\section{Funding}

This work is supported by National Natural Science Foundation of China (21903007), Young Thousand Talents Program (110532103), Beijing Normal University Startup funding (312232102), Beijing Municipal Science \& Technology Commission (No. Z191100000819002), and the Fundamental Research Funds for the Central Universities (310421109).

\section{References}

1. Pan, L. et al. Mechano-regulated metal-organic framework nanofilm for ultrasensitive and anti-jamming strain sensing. Nat. Commun. 9, 3813 (2018).

2. Lipomi, D. J. et al. Skin-like pressure and strain sensors based on transparent elastic films of carbon nanotubes. Nat. Nanotechnol. 6, 788-792 (2011).

3. Zhang, B. et al. Stretchable Conductive Fibers Based on a Cracking Control Strategy for Wearable Electronics. Adv. Funct. Mater. 28, 1801683 (2018).

4. Kang, S. et al. Bioresorbable silicon electronic sensors for the brain. Nature. 530, 71-76 (2016).

5. Wan, C. et al. An Artificial Sensory Neuron with Tactile Perceptual Learning. Adv. Mater. 30, 1801291 (2018).

6. Wan, C. et al. Artificial Sensory Memory. Adv. Mater. 32, 1902434 (2020). 
7. Wang, S. et al. Skin electronics from scalable fabrication of an intrinsically stretchable transistor array. Nature. 555, 83-88 (2018).

8. Kabiri Ameri, S. et al. Graphene Electronic Tattoo Sensors. Acs Nano. 11, 7634-7641 (2017).

9. Fernández, M. \& Pallás-Areny, R. Ag-AgCl electrode noise in high-resolution ECG measurements. Biomedical instrumentation \& technology. 34, 125-130 (2000).

10. Huigen, E., Peper, A. \& Grimbergen, C. A. Investigation into the origin of the noise of surface electrodes. Medical and Biological Engineering and Computing. 40, 332-338 (2002).

11. Leleux, P. et al. Ionic Liquid Gel-Assisted Electrodes for Long-Term Cutaneous Recordings. Adv. Healthc. Mater. 3, 1377-1380 (2014).

12. Green, R. M. et al. Benefits, shortcomings, and costs of EEG monitoring. Ann. Surg. 201, 785-792 (1985).

13. Nawrocki, R. A. et al. Self-Adhesive and Ultra-Conformable, Sub-300 nm Dry Thin-Film Electrodes for Surface Monitoring of Biopotentials. Adv. Funct. Mater. 28, 1803279 (2018).

14. Sekine, S., Ido, Y., Miyake, T., Nagamine, K. \& Nishizawa, M. Conducting Polymer Electrodes Printed on Hydrogel. J. Am. Chem. Soc. 132, 13174-13175 (2010).

15. Sasaki, M. et al. Highly Conductive Stretchable and Biocompatible Electrode-Hydrogel Hybrids for Advanced Tissue Engineering. Adv. Healthc. Mater. 3, 1919-1927 (2014).

16. Abidian, M. R. \& Martin, D. C. Multifunctional Nanobiomaterials for Neural Interfaces. Adv. Funct. Mater. 19, 573-585 (2009).

17. Liu, Y. et al. Soft and elastic hydrogel-based microelectronics for localized low-voltage neuromodulation. Nature Biomedical Engineering. 3, 58-68 (2019).

18. Keplinger, C. et al. Stretchable, Transparent, Ionic Conductors. Science. 341, 984 (2013).

19. Liu, Y. et al. Soft conductive micropillar electrode arrays for biologically relevant electrophysiological recording. Proceedings of the National Academy of Sciences, (2018).

20. Lee, W. H. et al. Control of Graphene Field-Effect Transistors by Interfacial Hydrophobic Self-Assembled Monolayers. Adv. Mater. 23, 3460-3464 (2011).

21. Schwierz, F. Graphene transistors. Nat. Nanotechnol. 5, 487-496 (2010).

22. Bunch, J. S. et al. Impermeable Atomic Membranes from Graphene Sheets. Nano Lett. 8, 2458-2462 (2008).

23. Lee, C., Wei, X., Kysar, J. W. \& Hone, J. Measurement of the Elastic Properties and Intrinsic Strength of Monolayer Graphene. Science. 321, 385 (2008).

24. Lee, W. H. et al. Surface-Directed Molecular Assembly of Pentacene on Monolayer Graphene for High-Performance Organic Transistors. J. Am. Chem. Soc. 133, 4447-4454 (2011).

25. Balandin, A. A. Thermal properties of graphene and nanostructured carbon materials. Nat. Mater. 10, 569-581 (2011).

26. Yu, Q. et al. Control and characterization of individual grains and grain boundaries in graphene grown by chemical vapour deposition. Nat. Mater. 10, 443-449 (2011).

27. Chen, J., Jang, C., Xiao, S., Ishigami, M. \& Fuhrer, M. S. Intrinsic and extrinsic performance limits of graphene devices on SiO2. Nat. Nanotechnol. 3, 206-209 (2008).

28. Liu, N. et al. Universal Segregation Growth Approach to Wafer-Size Graphene from Non-Noble Metals. Nano Lett. 11, 297-303 (2011).

29. Vosgueritchian, M., Lipomi, D. J. \& Bao, Z. Highly Conductive and Transparent PEDOT:PSS Films with a Fluorosurfactant for Stretchable and Flexible Transparent Electrodes. Adv. Funct. Mater. 22, 421-428 (2012).

30. Kim, K. et al. Ultrathin Organic Solar Cells with Graphene Doped by Ferroelectric Polarization. Acs Appl. Mater. Inter. 6, 32993304 (2014).

31. Fan, B., Mei, X. \& Ouyang, J. Significant Conductivity Enhancement of Conductive Poly(3,4ethylenedioxythiophene):Poly(styrenesulfonate) Films by Adding Anionic Surfactants into Polymer Solution. Macromolecules. 41, 5971-5973 (2008).

32. Wang, Y. et al. A highly stretchable, transparent, and conductive polymer. Science Advances. 3, e1602076 (2017). 
33. Ouyang, J. "Secondary doping" methods to significantly enhance the conductivity of PEDOT:PSS for its application as transparent electrode of optoelectronic devices. Displays. 34, 423-436 (2013).

34. Ouyang, J., Chu, C. W., Chen, F. C., Xu, Q. \& Yang, Y. High-Conductivity Poly(3,4-ethylenedioxythiophene):Poly(styrene sulfonate) Film and Its Application in Polymer Optoelectronic Devices. Adv. Funct. Mater. 15, 203-208 (2005).

35. Kee, S. et al. Controlling Molecular Ordering in Aqueous Conducting Polymers Using lonic Liquids. Adv. Mater. 28, 8625-8631 (2016).

36. Nawrocki, R. A. et al. Self-Adhesive and Ultra-Conformable, Sub-300 nm Dry Thin-Film Electrodes for Surface Monitoring of Biopotentials. Adv. Funct. Mater. 28, 1803279 (2018).

\section{Figures}

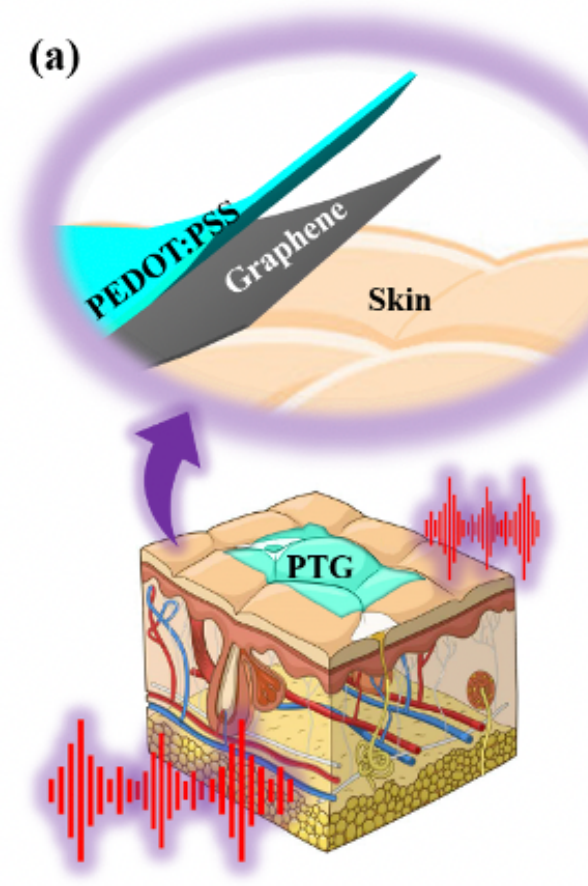

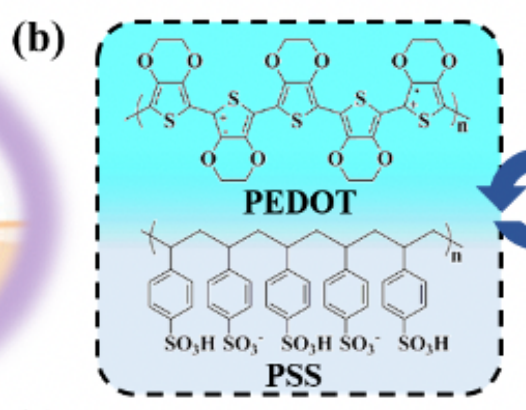

(c)

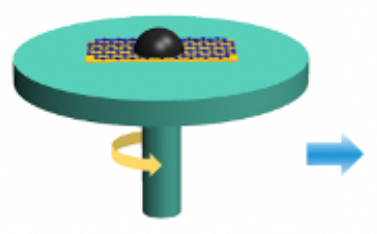

Spin-coationg

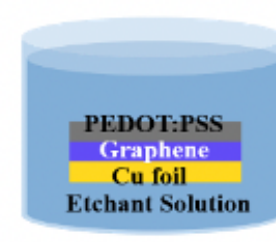

Etch-Cu
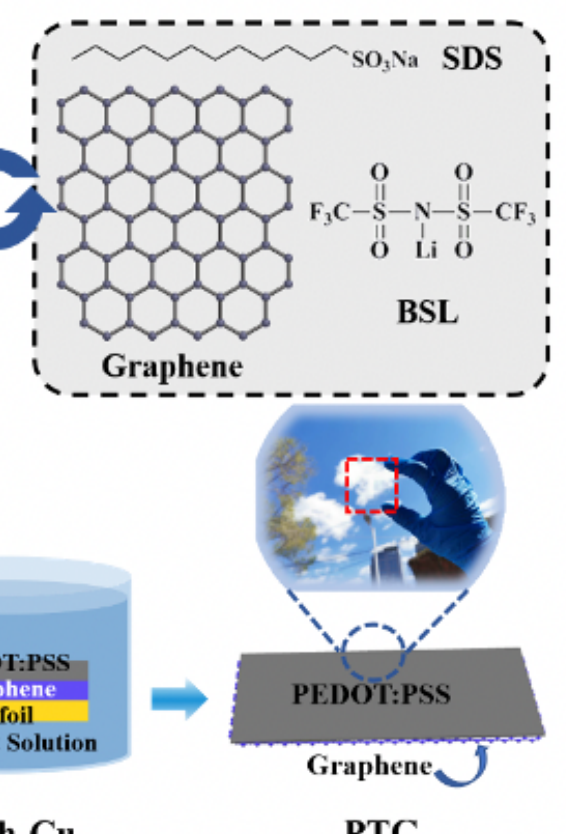

PTG

\section{Figure 1}

Fabrication of PTG as a skin electrode. (a) Schematic illustration of PTG as a skin electrode for electrophysiology. (b) Chemical structure of PEDOT:PSS, graphene, SDS, and BSL. (c) Schematic illustration of the fabrication process of the double-layer stacked structure of PEDOT:PSS/graphene. Inset is a transparent PTG held on a plastic substrate. 
(a)

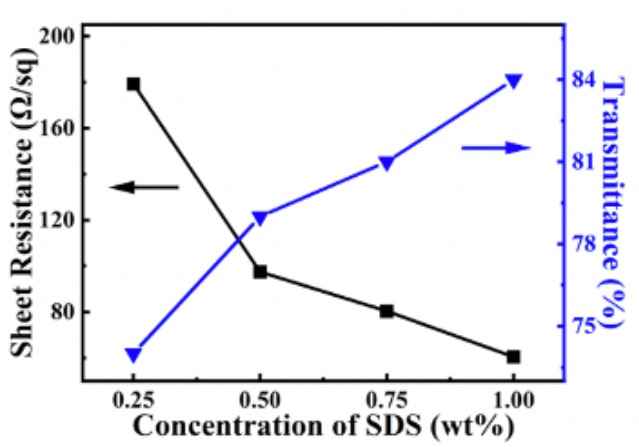

(c)

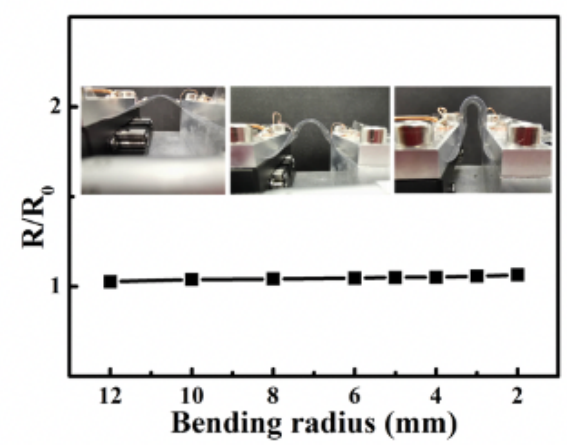

(e)

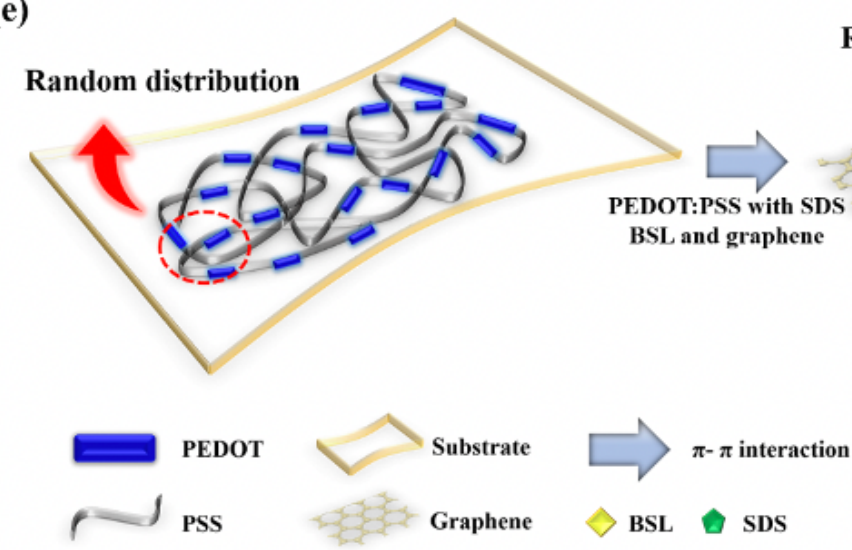

(b)

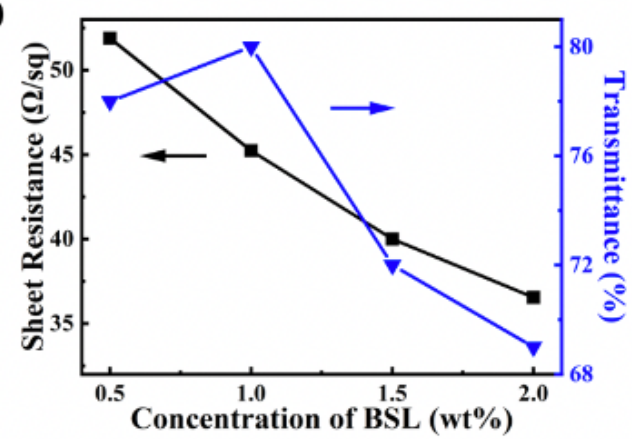

(d)

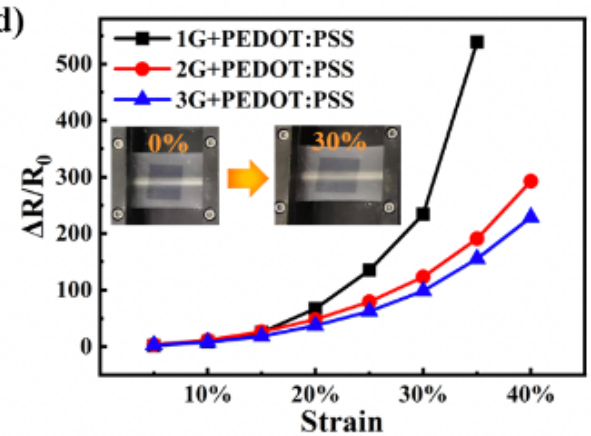

Figure 2

Optoelectronic performances of PTG. $(a, b)$ Sheet resistances and transmittances (at $\lambda=550 \mathrm{~nm}$ ) of PTG films at various SDS (a) and BSL (b, $1.0 \mathrm{wt} \%$ SDS) concentrations when forming PEDOT:PSS carrier layer. (c) Normalized resistance changes of PTGs on PET at bending radius from $12 \mathrm{~mm}$ to $2 \mathrm{~mm}$, and inset photographs showed PTGs on PET at different bending degrees. (d) Normalized resistance changes of PTGs on elastic SEBS at various strains. PTGs were made by PEDOT:PSS transferred mono-, bi-, and tri-layer graphene. (e) Schematic illustration of the synergistic enhancement in electrical conductivity by $\pi-\pi$ interaction and charge delocalization between graphene and PEDOT:PSS. 
(a)

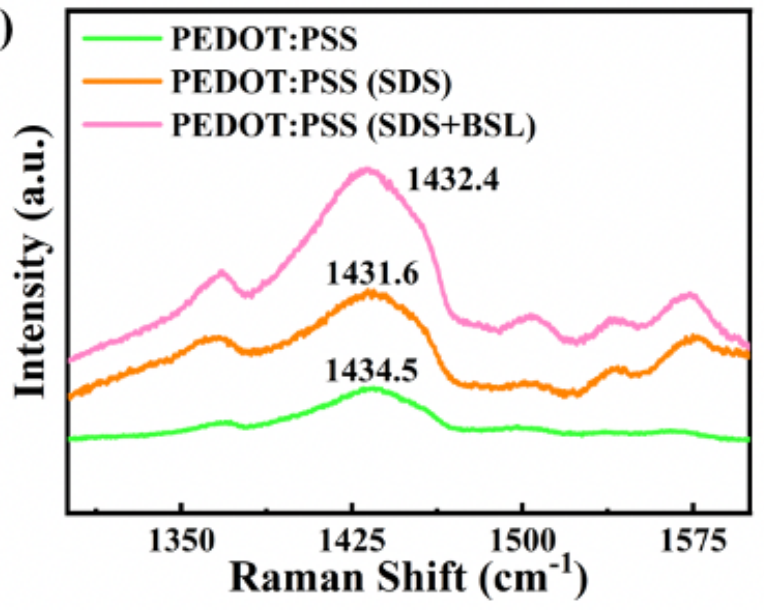

(c)

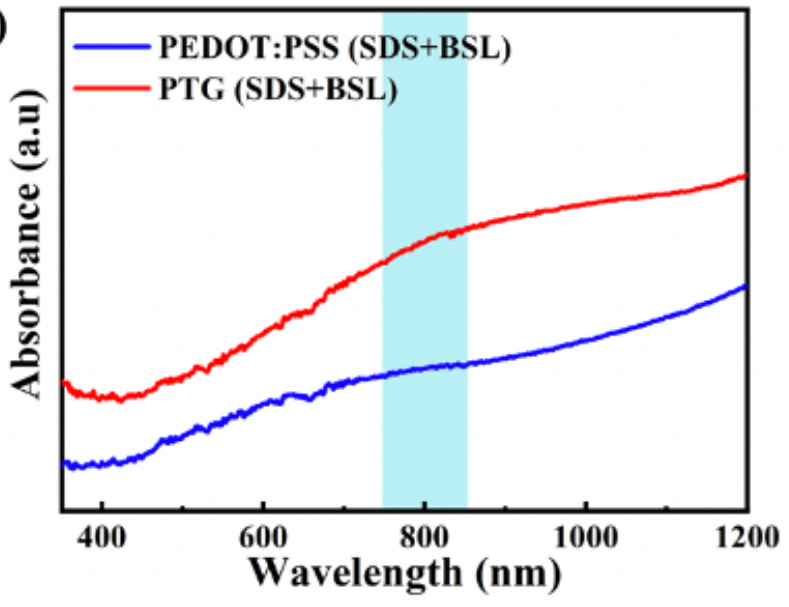

(b)

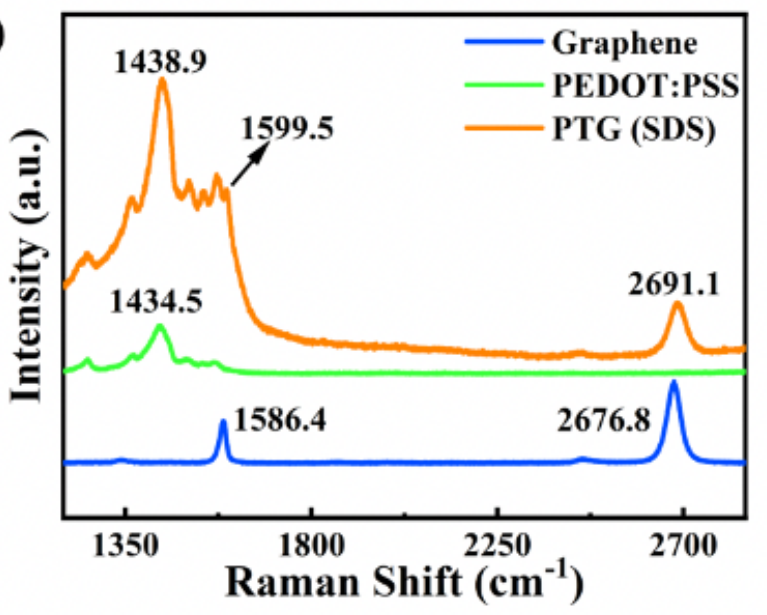

(d)

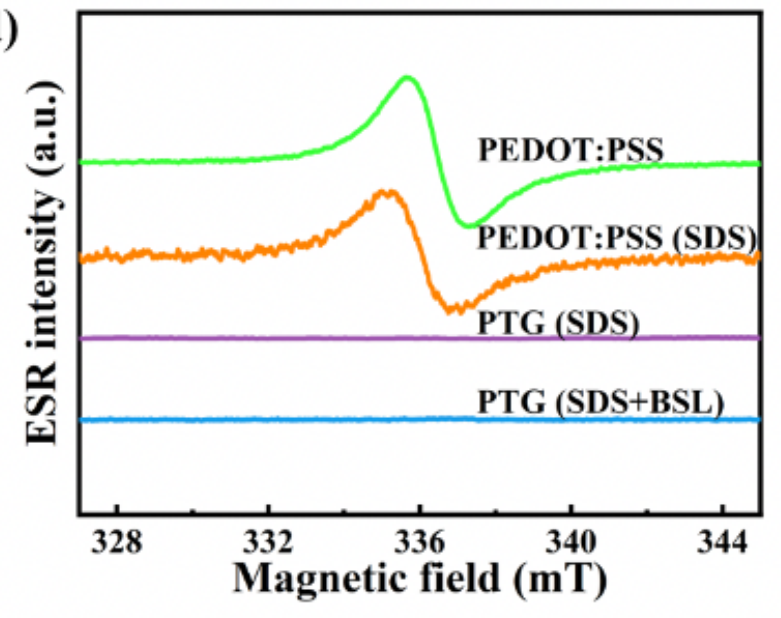

Figure 3

Synergistic enhancement between PEDOT:PSS and graphene. (a) Raman spectra of pure PEDOT:PSS films with different additives.

(b) Comparison of Raman spectra of graphene, pure PEDOT:PSS and PTG films. (c) UV-vis-NIR spectra of pure PEDOT:PSS and PTG films. (d) ESR spectra of various pure PEDOT:PSS and PTG films. 
(a)
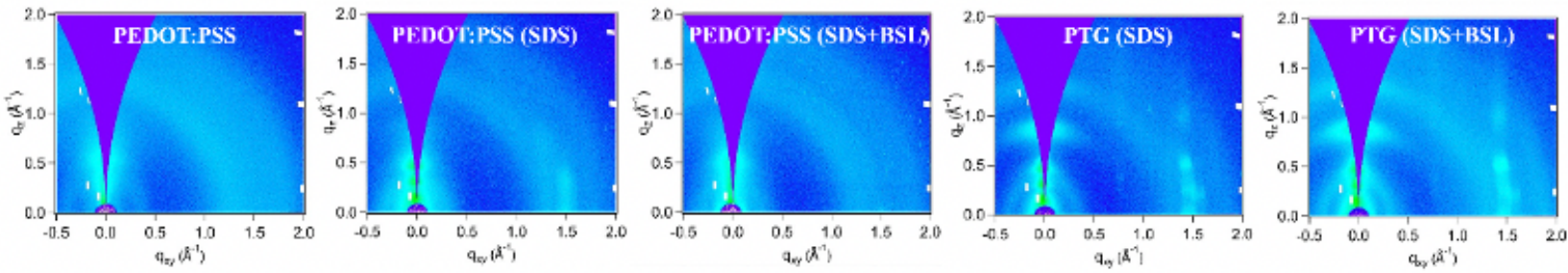

(b)
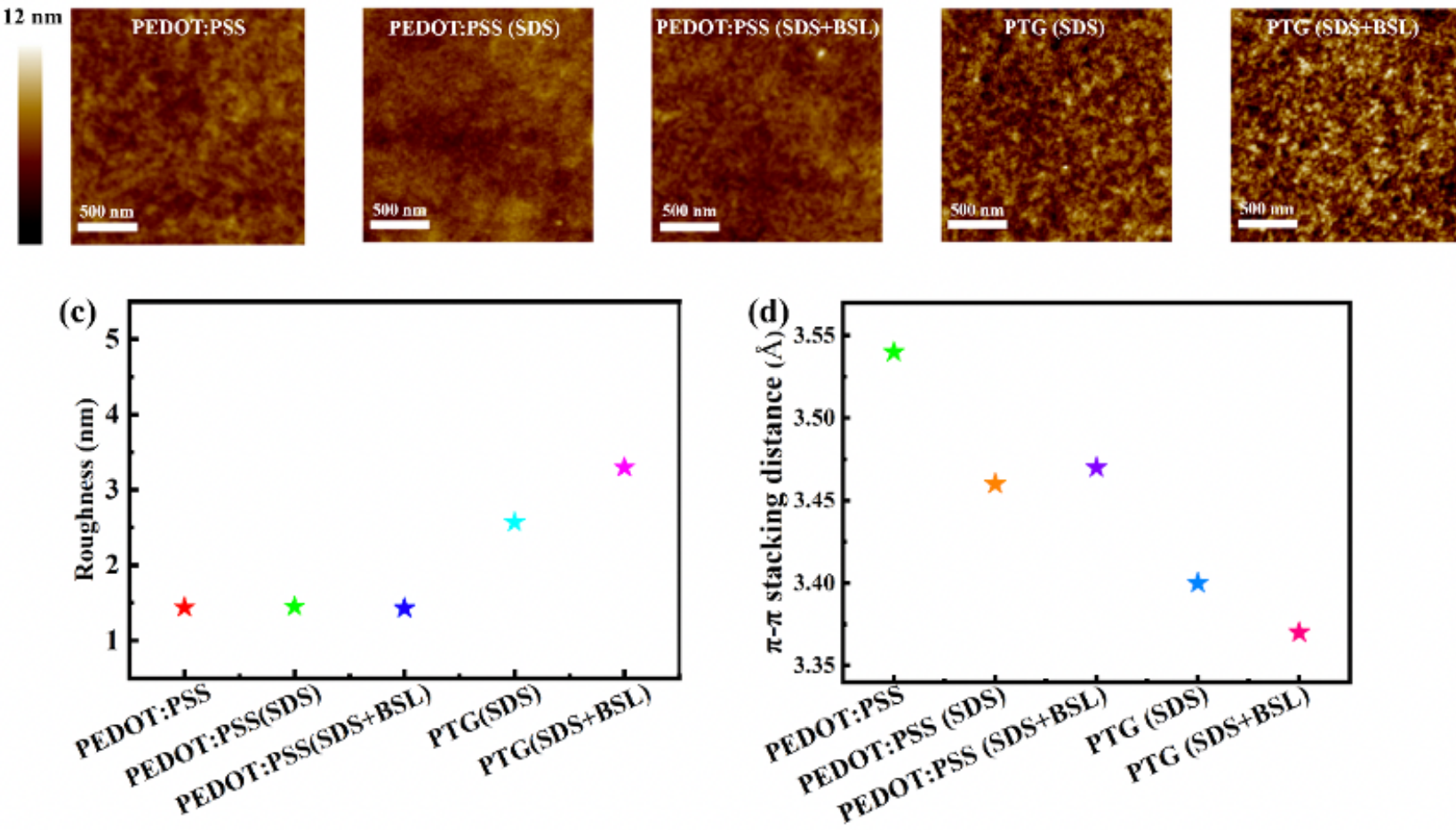

Figure 4

Highly ordered PEDOT in PTGs. (a) 2D GIWAXS patterns of pure PEDOT:PSS, PEDOT:PSS (SDS), PEDOT:PSS (SDS+BSL), PTG (SDS), and PTG (SDS+BSL). (b)AFM height images of various films. (c, d) Roughness (c) and $\pi-\pi$ stacking distances (d) of above films. 

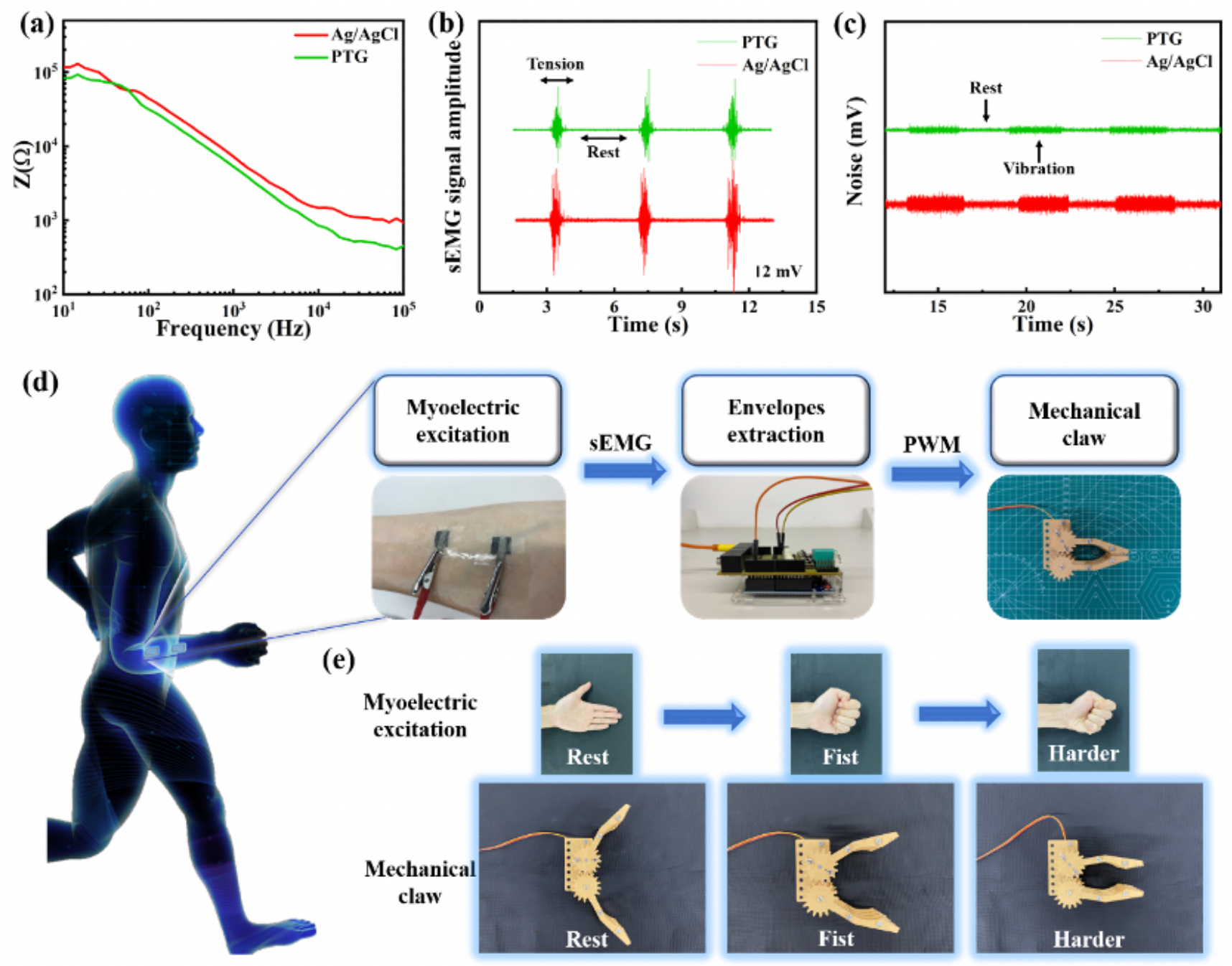

\section{Figure 5}

Electrophysiological signal detection of PTG dry electrodes. (a) The impedance analysis of PTG (green) and Ag/ AgCl electrodes (red). (b) sEMG measured by PTG and Ag/AgCl electrodes. (c) Motion artifact behavior of PTG and Ag/AgCl electrodes. (d) Schematic illustration of PTG electrodes on the arm for sEMG detection and robotic claws controlling process. Firstly the sEMG signals were extracted by PTG electrodes and then converted to Pulse Width Modulation (PWM) pulses to drive the servo motor. (e) The mechanical claws controlled by muscular excitation-contraction of human grip at various strengths.

\section{Supplementary Files}

This is a list of supplementary files associated with this preprint. Click to download.

- video.mp4

- SIUltraConformalSkinElectrodeswithSynergisticallyEnhancedConductivityforLowMotionArtifactEpidermalElectrophysiology.docx 